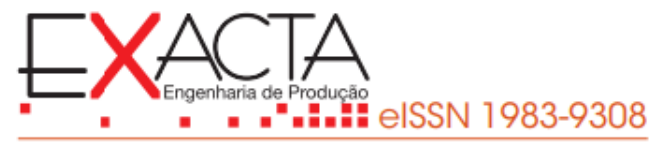

\title{
GESTÃO DO CONHECIMENTO E PRÁTICAS DE EXPLICITAÇÃO DE TÁCITO PARA EXPLÍCITO: UMA REVISÃO SISTEMÁTICA DA LITERATURA DOS ÚLTIMOS 20 ANOS
}

\section{KNOWLEDGE MANAGEMENT AND PRACTICES OF EXPLICITATION OF TACIT TO EXPLICIT: A SYSTEMATIC LITERATURE REVIEW OF LAST 20 YEARS}

Recebido em: 19 nov. 2019

Aprovado em: 16 ago. 2020

Versão do autor aceita publicada online: 16 ago. 2020

Publicado online: 19 maio 2021

\section{Como citar esse artigo - American Psychological Association (APA):}

Alves, R. R., \& Campos, F. C. (2021, out./dez.). Gestão do conhecimento e práticas de explicitação de tácito para explícito: uma revisão sistemática da literatura dos últimos 20 anos. Exacta. 19(4), 911932. https://doi.org/10.5585/exactaep.2021.16057.

Submeta seu artigo para este periódico $\beta$

Dados Crossmark 


\title{
GESTÃO DO CONHECIMENTO E PRÁTICAS DE EXPLICITAÇÃO DE TÁCITO PARA EXPLÍCITO: UMA REVISÃO SISTEMÁTICA DA LITERATURA DOS ÚLTIMOS 20 ANOS
}

\author{
KNOWLEDGE MANAGEMENT AND PRACTICES OF EXPLICITATION OF TACIT TO \\ EXPLICIT: A SYSTEMATIC LITERATURE REVIEW OF LAST 20 YEARS
}

Rafael Rodrigues Alves ${ }^{1}$

Fernando Celso de Campos

\author{
${ }^{1}$ Mestre. \\ Universidade Metodista de Piracicaba - UNIMEP. \\ Sta. Barbara d'Oeste, São Paulo - Brasil. \\ rafaelralves@outlook.com \\ 2 Pós-doutorado \\ Universidade Metodista de Piracicaba - UNIMEP. \\ Sta. Barbara d’Oeste, São Paulo - Brasil. \\ fernando.campos@unimep.br
}

Recebido em: 19 nov. 2019

Aprovado em: 16 ago. 2020
Resumo: Com o cenário competitivo cada vez mais agressivo as empresas passam por um momento delicado quando se trata da busca de mão de obra especializada, com isso, as companhias vêm adotando práticas inovadoras de gestão do conhecimento. Porém, se tratando de conhecimento tácito, ainda existem dúvidas sobre as melhores práticas de explicitação desse tipo de conhecimento. Assim, este trabalho tem o objetivo de realizar uma revisão sistemática da literatura no intuito de identificar e analisar as principais publicações com foco nas práticas de explicitação de conhecimento tácito, a fim de compreender a constituição deste tema no que tange as boas práticas adotadas pelas empresas e as respectivas perspectivas de pesquisa. Com o método de análise, busca-se em bases de dados internacionais, no período entre 1998 e abril-2019, a identificação de lacunas e tendências sobre o tema. Como resultado, elencou-se as principais práticas de transferência do tácito, sendo elas: por auxílio de softwares, por programa de mentoria e aplicação de metodologias de transferências de conhecimento, assim como foram encontradas lacunas como: a falta de indicadores para mensurar a transferência do conhecimento e o alto tempo do ciclo total de transferência de conhecimento.

Palavras-chave: Gestão de conhecimento. Conhecimento explícito. Conhecimento tácito. Explicitação. Revisão sistemática da literatura.

Abstract: The most valuable resources that company have is the organizational knowledge and the scenario is each day more competitive, to find more specialized manpower, and with that companies are adopting innovative practices about knowledge management. But facing the tacit knowledge there are doubts about the practices of explicitation of that kind of knowledge. This paper aims to perform a systematic literature review based on the best practices of explicitation of tacit knowledge to support companies to implement a more efficiency and effective knowledge management. The method of analysis adopts a research in three different databases for the period between 1998 and April 2019, to identify gaps and tendencies about the theme. As results there are finds of the main practices of tacit knowledge transfer being them with the support of software, mentoring programs and the application of the methodologies of knowledge transfer, as well as found some gaps as the lack of indicators to measure the knowledge transfer and the high time the explicitation succeed in this kind of knowledge.

Keywords: Knowledge management. Explicit knowledge. Tacit knowledge. Explicitation. Systematic literature review. 


\section{Introdução}

No recente cenário produtivo, as empresas têm focado nas atividades que garantam maior qualidade em seus produtos, atendendo as especificações dos seus clientes, satisfazendo-os. Apesar de altos investimentos em novas tecnologias, ainda depende-se da intervenção humana no ambiente industrial. Com isso a mão de obra experiente torna-se um diferencial competitivo, além disso, existem riscos que as companhias enfrentam no que diz respeito a pessoas, tais como: aposentadorias, ausências de longo prazo devido a assuntos médicos, perda de pessoas para outras companhias. Frente a isso, é necessária a adoção de práticas voltadas à gestão do conhecimento, tanto explícito quanto tácito. Porém, tratando-se das práticas de gestão de conhecimento explícito, estas estão consolidadas nas companhias pois, segundo Ribeiro (2012), é um conhecimento que pode ser articulado e gerenciado facilmente. Já para Nonaka e Takeuchi (1995), o conhecimento tácito é o contrário do conhecimento explícito. Este último pode ser definido como linguagens formais e que, geralmente, são expressos em equações matemáticas, manuais, entre outros. Para o conhecimento tácito, Badaracco (1991) o define como um conhecimento intuitivo, não articulado e até mesmo inexpressável.

Porém, realizar a gestão do conhecimento tácito não é tarefa fácil. Sendo assim é necessário que haja exploração aprofundada de estudos sobre o tema, identificando os pontos e práticas mais relevantes, os destaques que os principais autores e periódicos tem realizado, de modo a classificar as dificuldades enfrentadas pelas empresas e auxiliar na explicitação desse tipo de conhecimento.

Para identificação dessas publicações, foi adotado como método a revisão sistemática da literatura (RSL) que exerce um papel importante para a produção científica, pois, segundo Ramy et al. (2018) as reflexões da literatura permitem que sejam minimizadas repetições e criam pontos de partida para avanços futuros na teoria e na prática. O método de Jesson, Matherson e Lacey (2011) permite o pesquisador identificar, avaliar e interpretar toda pesquisa já disponível e relevante para área temática em particular. Já o método de Hood e Wilson (2001) analisa a descrição do estudo da ciência, incluindo crescimento, estrutura, inter-relações e produtividade de uma certa temática de pesquisa. De acordo com Al-Tabbaa e Ankrah (2016), a RSL no campo da gestão vêm sendo cada vez mais adotada por se tratar de um processo confiável e rigoroso que reduz o viés subjetivo e riscos de negligenciar pesquisas relevantes.

Por este motivo, este trabalho tem como objetivo identificar e analisar as principais publicações, por intermédio do método de RSL, tendo como principal foco as práticas de explicitação do conhecimento tácito, a fim de compreender a constituição deste tema no que tange as boas práticas adotadas pelas empresas e as respectivas perspectivas de pesquisa.

Assim, o presente trabalho está estruturado em seis seções passando por uma seção introdutória, seguida de uma revisão da literatura dos principais assuntos abordados, logo após uma 
seção com a apresentação do método de pesquisa, dos resultados e discussão, lacunas e tendências, concluindo com as principais contribuições e achados encontrados sobre o tema.

\section{Revisão da literatura}

Nesta seção explora-se uma revisão da literatura de três conceitos: gestão do conhecimento, explicitação de conhecimento e do conhecimento tácito, a fim de identificar as principais referências dos temas abordados.

\subsection{Gestão do conhecimento}

A gestão de conhecimento, de modo geral, é um assunto amplamente abordado e consolidado em diversos segmentos, garantindo um considerável grau de importância para o crescimento de vantagem competitiva para empresas que adotam práticas de captura e compartilhamento de conhecimento (PULIC, 1998).

Pode ser definida como um processo de criar, adquirir, transferir, documentar e aplicar o conhecimento (NONAKA \& TAKEUCHI, 1995).

Em uma visão voltada a estratégia de negócios das companhias, o conhecimento foi identificado como um dos recursos mais importantes e a principal fonte de criação de valor (TEECE \& AL-AALI, 2011).

Mas, para explorar a gestão de conhecimento, é necessário um passo adiante, no qual pode-se classificar os tipos de conhecimentos que, conforme Polanyi (1969), o conhecimento explícito pode ser traduzido por linguagens formais, muitas vezes expresso, de modo sistemático, em equações matemáticas, manuais, entre outras formas e o conhecimento tácito, expressado como um conhecimento informal, tendo vínculo aos sentimentos, à percepção individual, à capacidade de expressão corporal, convicções e perspectivas de quem detém deste conhecimento.

Deste modo, segundo Nonaka e Takeuchi (1995), o conhecimento explícito é mais facilmente difundido, embora Polanyi (1969) enfatize que "o conhecimento explícito deve apoiar-se em ser tacitamente entendido e aplicado" o conhecimento explícito é taxado como um conhecimento fácil de transferir ou até mesmo explicado ou roteirizado.

Arling e Chun (2011) definem o conhecimento explícito como conhecimento codificável, ou seja, que pode ser organizado e comunicado por meio formalizado ou figurado. E conforme Beal (2014) esse tipo de conhecimento é definido como dados que podem ser entendidos por meio de registros. Em outras palavras, é um conhecimento que pode ser comunicado por meio de processos, figuras ou fórmulas.

Já o conhecimento tácito, conforme observado por Gill (2000), traz a dificuldade na transformação do conhecimento em algo que possa ser utilizado ou disseminado entre outras pessoas. 
O autor enfatiza que a externalização do conhecimento tácito pode gerar conflito de informações, pois, muitas vezes, o conhecimento tácito para quem o detém está intrínseco nas ações do dia a dia. Essa dificuldade de entender, transcrever e aplicar o conhecimento tácito, segundo Machado (2002), se dá por ser algo que as pessoas são capazes de realizar sem a consciência da ação. De maneira diferente, Augier e Vendelo (1999) enfatizam que o conhecimento tácito é totalmente individual, tornando a sua difusão impossível, sendo atingível apenas por experiência interpessoal.

Já para Nonaka et al. (2014), tanto o conhecimento tácito quanto o explícito estão interligados de alguma forma, afirmando que todo conhecimento explícito contém aspectos do conhecimento tácito. Ou seja, é necessário que exista a interação entre pessoas no ato de compartilhamento ou criação de conhecimento, criando uma cultura de disseminação de conhecimento entre as pessoas envolvidas dentro da companhia.

De acordo com Choo e Neto (2010), o sucesso da gestão do conhecimento depende principalmente da mudança de comportamento das pessoas.

Embora existam diversos níveis de compartilhamento do conhecimento, a maneira mais assertiva é realizando a transferência do conhecimento de um indivíduo para outro, pois deve-se considerar a similaridade e a relação entre pessoas, sendo características do processo de transferência (ZHANG \& JIANG, 2015).

O ambiente ideal para a gestão do conhecimento, segundo Nonaka e Takeuchi (1995), é definido por um processo de mudança ou transferência do conhecimento entre pessoas, equipes da organização, ilustrado pelo modelo SECI (Figura 1), no qual: a Socialização se dá pela transferência do conhecimento tácito por meio do compartilhamento da experiência vivida; a Externalização envolve a mudança do conhecimento tácito para o explícito, externalizando o conhecimento tácito que está internalizado; a Combinação é o processo que combina vários tipos de conhecimento explícito para criar um novo conhecimento explícito; e a Internalização envolve o desenvolvimento do conhecimento tácito por meio da experiência. 
Figura 1

Representação do modelo SECI ou espiral do conhecimento

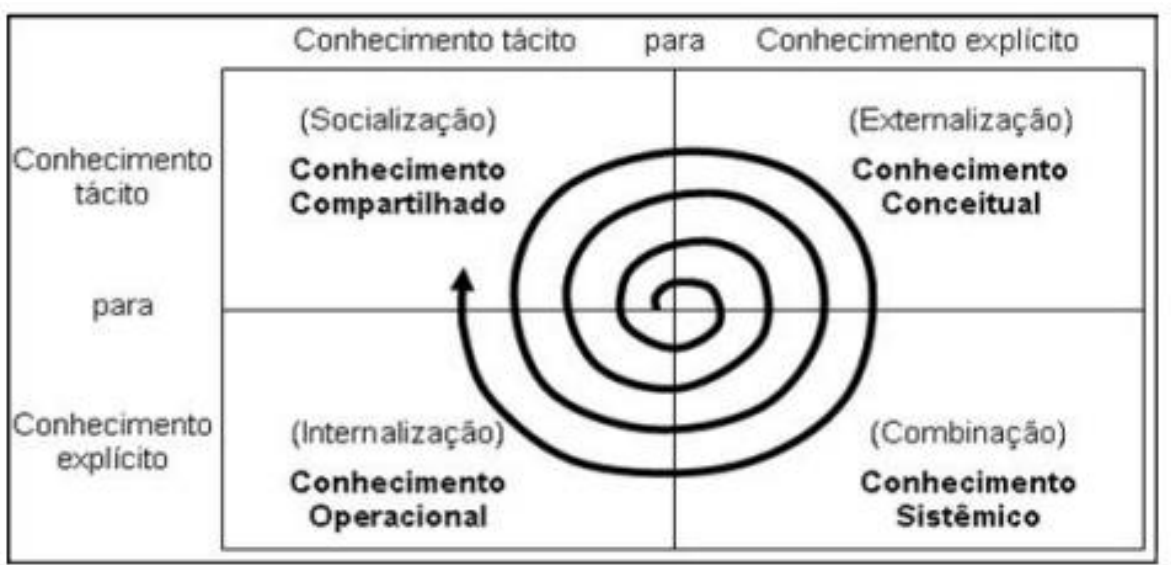

Fonte: Nonaka e Takeuchi (2008).

Uma das ferramentas adotadas pelas empresas para gestão do conhecimento é o incentivo-em compartilhar o conhecimento entre indivíduos, podendo gerar novas ideias e, consequentemente, novos conhecimentos (LEON, 2017). Já, de acordo com Choi et al. (2008), quando o conhecimento é criado e posteriormente disseminado dentro da companhia, tem potencial de contribuir com a capacidade de solução de problemas de forma mais assertiva e inovadora.

\subsection{Conhecimento tácito}

O conhecimento tácito é abordado há décadas, conforme evidenciado anteriormente (Polanyi, 1983, 1966). É uma referência a um tipo de conhecimento que foi desenvolvido com o tempo, ou seja, pela experiência adquirida ao longo de anos e que não pode ser transmitido por meio de simples palavras (Ribeiro, 2012), textos, manuais, entre outros. É como se houvesse um limitador na fala dos indivíduos ou da "codificação" que definiria ou distinguiria o que é tácito do que é explícito.

Wittgenstein (1976) aborda-o conhecimento tácito como sendo socialmente acordado, modificado e posteriormente transmitido. Para Kuhn (1996), conhecimento tácito é resultado de um "acordo social" independentemente do tipo do conhecimento, o qual terá influência com o tempo, de acordo com a sua evolução. Collins (2007) define como "conhecimento tácito coletivo". Entretanto, Ribeiro (2012) afirma que o conceito do tácito também é usado em dois sentidos diferentes: o primeiro para identificar as habilidades humanas, por exemplo andar de bicicleta. O segundo se refere a práticas "pressupostas" que indivíduos aprendem, sem se dar conta, no processo de tornarem-se membros de um grupo, um exemplo é quando novatos fazem algo que pessoas experientes nunca fariam, mas que, apenas percebem isso quando, inesperadamente, os veem quebrando regras "pressupostas". 


\subsection{Explicitação do conhecimento tácito}

A explicitação do conhecimento surge da necessidade de segregar o conhecimento adquirido, ao longo dos anos, pelo ser humano, seja este explícito ou tácito. Referindo-se ao conhecimento explícito, a explicitação se dá por meio de leituras de manuais, textos, procedimentos, entre outros. Já o conhecimento tácito, segundo Dutta (1997) e Wagner \& Sternberg (1985), é intangível, pois representa a intuição, insights, crenças e experiências.

Embora a gestão de conhecimento tácito seja um assunto amplamente explorado na literatura, ainda surgem lacunas quanto às melhores práticas de explicitação deste tipo de conhecimento, devido à dependência da interação do indivíduo que detém o conhecimento com o tipo de explicitação. Como observado por Choi \& Lee (2002), cria-se o conhecimento quando o mesmo muda da forma tácita para explicita. Já para Wiig (1993), o conhecimento é adquirido por meio do intelecto do indivíduo e da análise prévia do conhecimento a ser adquirido e, posteriormente, se concretiza com tentativas e erros.

Já para Szulanski (1996), a transferência do conhecimento é construída por trocas de informações individuais, sendo mais assertiva quando o grau de intimidade entre tutor e aprendiz for mais elevado. Da mesma forma, de acordo com Cumming (2004), a explicitação do conhecimento tem sido demonstrada por meio da interação do time e pela realização de trabalhos em conjunto. Além disso, há melhoria no desempenho da equipe, bem como a fomentação à geração de novos conhecimentos por meio de ideias inovadoras geradas neste ciclo.

Desta forma, Kakabadse et al. (2001) afirmam que o conhecimento tácito só pode ser transmitido se houve a possibilidade de convertê-lo em palavras, números, ou imagens que sejam compreendidos por outros. Isso diverge das afirmações de Nonaka e Takeuchi (1995) sobre a máquina de pão japonês, para a qual o conhecimento tácito do mestre-padeiro foi convertido em explícito e transferido.

\section{Método de pesquisa}

Para o desenvolvimento deste trabalho, foi realizada uma revisão sistemática da literatura (RSL) que exerce papel importante para a produção científica, pois, segundo Ramy et al. (2018), as reflexões da literatura permitem que sejam minimizadas repetições e criam pontos de partida para avanços futuros na teoria e na prática.

Jesson, Matherson e Lacey (2011) afirmam que a RSL é um método que permite ao pesquisador identificar, avaliar e interpretar toda pesquisa já disponível e relevante para um tema específico.

Já Hood e Wilson (2001) citam que é um método de análise utilizado para descrever o estudo da ciência, incluindo crescimento, estrutura, inter-relações e produtividade de uma certa temática de pesquisa. e De acordo com Al-Tabbaa e Ankrah (2016), a RSL no campo da gestão vem sendo cada vez 
mais adotada por se tratar de um processo confiável e rigoroso, reduzindo o viés subjetivo e riscos de negligenciar pesquisas relevantes.

O processo de RSL foi conduzido em quatro estágios, conforme expressado pela Figura 2 e detalhados, respectivamente, na sequência.

\section{Figura 2}

Estrutura do método de revisão sistemática da literatura

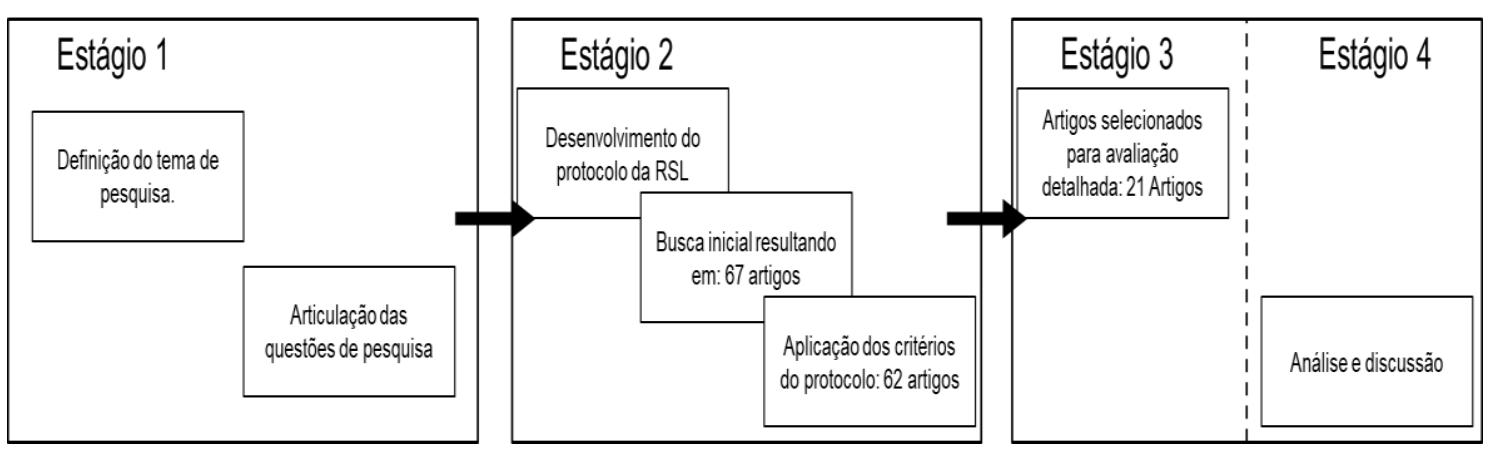

Fonte: Elaborado pelos próprios autores.

No primeiro estágio, os objetivos e as questões de pesquisa foram formulados. Baseado nisso, foram determinadas as palavras-chave, posteriormente apresentado no protocolo da RSL.

No segundo estágio, a fim de garantir o rigor e a confiabilidade desta RSL, adotou-se os protocolos sugeridos por Kitchenham (2007) e Tranfield et al. (2003). No protocolo de busca (Quadro 1), especificou-se um conjunto explícito de combinações de termos (strings de busca) para identificar, de forma assertiva, os artigos a serem incluídos. 


\section{Quadro 1}

Protocolo da RSL

\section{PROTOCOLO RSL - Explicitação de Conhecimento Tácito}

1) Background

2) Questões da Pesquisa

3) Estratégias de Pesquisa

4) Palavras-Chave combinadas

5) Critérios de Exclusão

6) Critérios de Inclusão

7) Estratégia para organização de dados

8) Cronograma
Visão geral das práticas de explicitação do conhecimento tácito.

I. Quais são as principais práticas de explicitação de conhecimento tácito?

II. Quais são os indicadores utilizados para medir a performance do conhecimento explicitado e posteriormente adquirido?

III. Existem padrões para gestão do conhecimento tácito?

Base de Dados: Science Direct, Scopus e Web of Science

("Knowledge management" AND "explicitation" AND tacit AND explicit)

I. Artigos que não são revisados por pares;

II. Artigos que não são publicados em periódicos;

III. Artigos publicados fora do período pré-estabelecido de 1998 a abril/2019;

IV. Artigos que não foram publicados nos idiomas: inglês ou português;

V. Quaisquer tipos de publicação que não sejam artigos.

1. Artigos identificados pelo processo "bola de neve";

2. Dos autores selecionados pelo processo "bola de neve" será avaliada a possível inclusão de artigos do mesmo se caso houver link entre os assuntos;

3. Artigos publicados no período pré-estabelecido de 1998 a abril/2019.

I. Utilização do Software MS Excel ${ }^{\circledR}$ e VOSviewer ${ }^{\circledR}$

Projeção da R.S.L. ter duração de 3 meses já incluso revisões.

Fonte: Elaborado pelos próprios autores.

Uma vez desenvolvido o protocolo da RSL, a pesquisa foi realizada em duas etapas: busca inicial na base de dados e aplicação dos critérios adotados nesse protocolo. A busca inicial concentrou-se nas bases de dados definidas no protocolo, bem como a string de busca adotada, resultando em 67 artigos. A aplicação dos critérios adotados no protocolo, conforme o Quadro 1, itens 5 e 6, resultaram em 62 artigos selecionados.

O terceiro estágio concentrou-se na análise dos 62 artigos selecionados.

A análise de cada artigo foi ampliada, realizando a leitura/interpretação dos títulos e resumos, incluindo as seções de introdução e conclusão.

Esta análise permitiu filtrar artigos de maior relevância relacionados ao tema da pesquisa.

Dessa forma, foram excluídos 42 artigos do escopo desta pesquisa. No final do estágio 3, chegou-se a 21 artigos para a análise completa.

No estágio 4, foram apresentadas as análises e discussões dos 21 artigos selecionados. A síntese será tratada na Seção 4. 
É preciso destacar que, para a realização das tabulações, bem como as análises dos artigos, foi utilizada uma planilha eletrônica (MS Excel $\left.{ }^{\circledR}\right)$. Para validar a amostra de artigos e proceder a análise de clusters, foi utilizado o software VOSviewer ${ }^{\circledR}$.

Com o desenvolvimento da RSL foi possível identificar alguns achados e tendências, o que possibilitou o desdobramento/detalhamento de aspectos da questão de pesquisa, norteando o estudo de caso: i) principais práticas explicitação de conhecimento tácito; ii) indicadores utilizados para medir a performance do conhecimento explicitado e posteriormente adquirido; iii) possíveis padrões para gestão do conhecimento tácito.

\section{Discussão dos resultados}

A partir da string de busca criada unindo as palavras-chave "Knowledge management", "explicitation", "tacit" e "explicit", um número de 67 artigos foram retornados e distribuídos nas bases de dados conforme ilustrado na Figura 3.

\section{Figura 3}

Total de artigos encontrados por base de dados

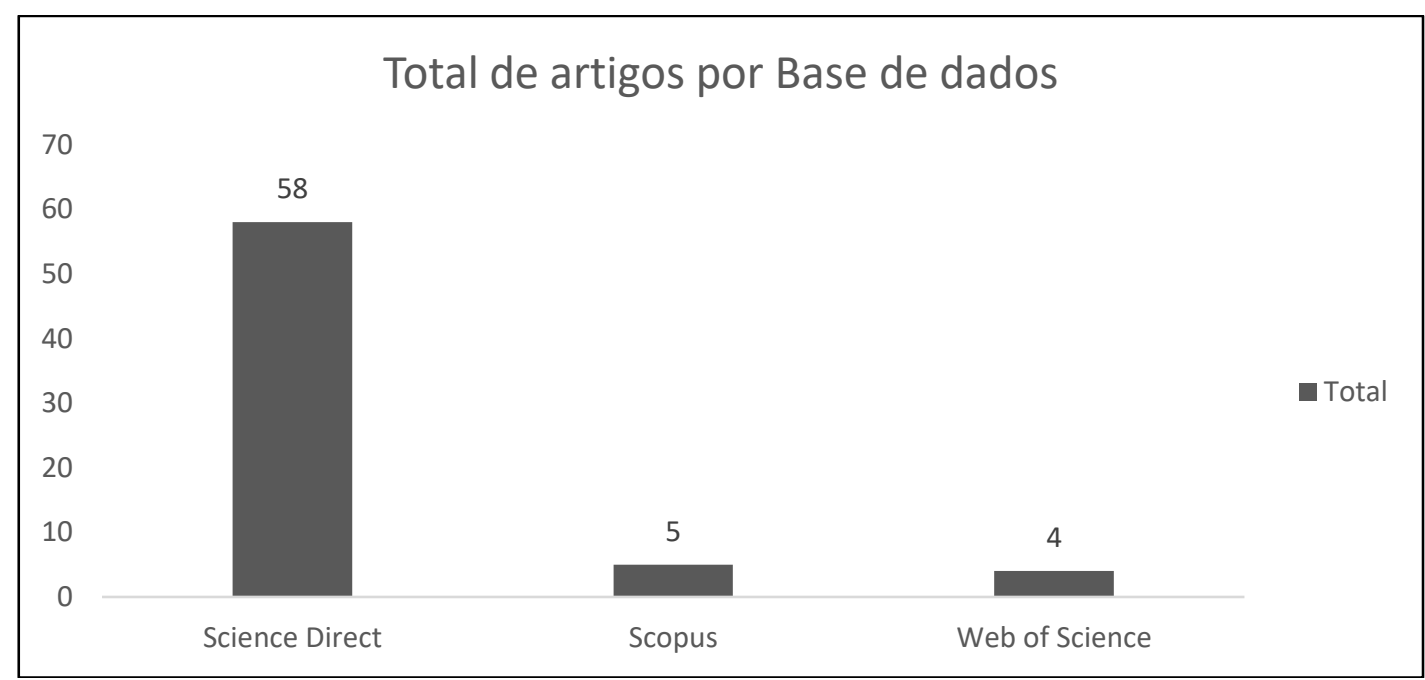

Fonte: Elaborado pelos autores.

Para validação da amostra, foi adotado o método de análise de clusters, por meio do software VOSviewer $^{\circledR}$, obtendo-se um agrupamento conforme ilustrado na Figura 4. 
Figura 4

Análise de clusters auxiliado pelo software VOSviewer ${ }^{\circledR}$

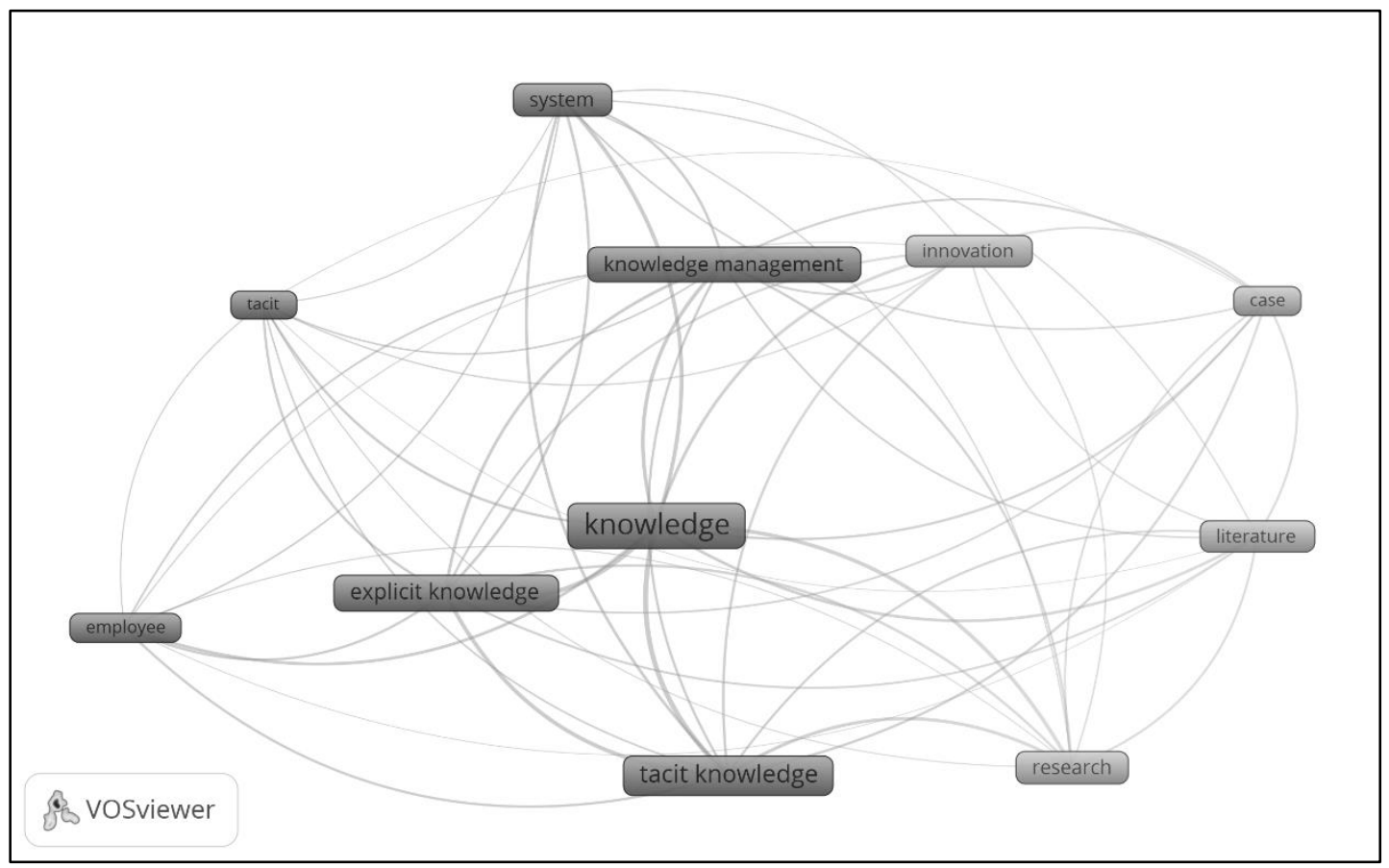

Fonte: Elaborado pelos autores.

Pode-se afirmar que as palavras-chaves que ficaram em evidência foram "knowledge" e "tacit knowledge", validando a amostra adotada para a análise.

Também foi adicionado um artigo por meio de análise das referências das principais pesquisas analisadas.

A partir desta amostra, foram aplicados os critérios do protocolo e realizada a análise dos artigos, resultando em 21 artigos relacionados ao escopo da pesquisa.

Desta forma, foi possível evidenciar as principais práticas de explicitação de conhecimento, conforme é-ilustrado no Quadro 2. Assim, foram realizadas as análises completas dos artigos para identificação das lacunas e tendências destas práticas de gestão do conhecimento. Estas análises são detalhadas na próxima seção. 


\section{Quadro 2}

Tipos de explicitação de conhecimento por autor

\begin{tabular}{|c|c|c|}
\hline $\begin{array}{l}\text { Práticas de } \\
\text { Explicitação }\end{array}$ & Descrição & Trabalhos Relacionados \\
\hline $\begin{array}{l}\text { Convivência / } \\
\text { Socialização }\end{array}$ & $\begin{array}{l}\text { Socialização ou convivência entre as } \\
\text { partes interessadas na gestão do } \\
\text { conhecimento. É efetivamente } \\
\text { considerado como tais práticas os } \\
\text { diálogos, coaching ou mentoring em } \\
\text { projetos, análises práticas ou execução } \\
\text { de trabalhos em conjunto. }\end{array}$ & $\begin{array}{l}\text { Olaisen, J., Revang, O. (2018) } \\
\text { Feng, W. J., et al. (2017) } \\
\text { Scaringella, L., Burtschell, F. (2017) } \\
\text { Serna E., et al. (2017)* } \\
\text { Belay, A. M., et al. (2016) } \\
\text { Oyemomi, O., et al. (2016)* } \\
\text { Chumg, H. F., et al. (2015) } \\
\text { Hau, Y. S., et al. (2013) } \\
\text { Xiang, X. Y., et al. (2013) } \\
\text { Yu, Y., et al. (2013) } \\
\text { Reychav, I. Weisberg, J. (2009) } \\
\text { Hall, R., Andriani, P. (2003) }\end{array}$ \\
\hline Softwares & $\begin{array}{l}\text { Softwares ou sistemas que auxiliam na } \\
\text { gestão do conhecimento considerando } \\
\text { softwares interativos nos quais os } \\
\text { usuários consigam se comunicar ou } \\
\text { somente de caráter descritivo. }\end{array}$ & $\begin{array}{l}\text { Serna E., et al. (2017)* } \\
\text { Lin, Y. C., Lee, H. Y. (2012) } \\
\text { Lin, Y. C. (2008) } \\
\text { Tserng, H. P., Lin, Y. C. (2004) } \\
\text { Woo, J. H., et al. (2004) } \\
\text { Noh, J. B., et al. (2000) }\end{array}$ \\
\hline Salas / Espaços & $\begin{array}{c}\text { Salas ou espaços específicos para } \\
\text { realização de socializações ou } \\
\text { interações. São considerados esses } \\
\text { ambientes as Fab labs, Ba's ou salas de } \\
\text { jogos. }\end{array}$ & $\begin{array}{l}\text { Maravilhas, S., Martins, J. (2019) } \\
\text { Oyemomi, O., et al. (2016)* } \\
\text { Desouza, K. C. (2003) }\end{array}$ \\
\hline Treinamentos & $\begin{array}{l}\text { Treinamentos teóricos ou práticos, } \\
\text { sendo considerado os mentores dos } \\
\text { treinamentos a pessoa que detém o } \\
\text { conhecimento a ser gerido. }\end{array}$ & $\begin{array}{l}\text { Tyagi, S., et al. (2015) } \\
\text { Zhao, J., et al. (2014) }\end{array}$ \\
\hline
\end{tabular}

Nota: $(*)$ Trabalhos que citam mais de um tipo de explicitação de conhecimento tácito.

Fonte: Elaborado pelos autores.

\section{Lacunas e tendências}

A amostra extraída desta pesquisa revelou trabalhos de diversas áreas. Embora o gerenciamento de conhecimento seja um assunto amplamente explorado, ainda é evidente uma lacuna por parte das publicações relacionadas às práticas e métodos de explicitação do conhecimento tácito, caracterizando as pesquisas que tratam a gestão do conhecimento em um modo generalizado, focando não apenas em um tipo de conhecimento.

Segundo Woo et al. (2004), a gestão do conhecimento tácito é importante para o sucesso da companhia, e deve ser gerenciado separadamente do conhecimento explícito, por meio de estratégias diferentes. Já como foi observado por Olaisen e Revang (2018), não é possível armazenar ou reusar o conhecimento tácito, em outras palavras, não pode ser documentado. Diferentemente do conhecimento explícito, que pode ser facilmente gerenciado e transcrito em procedimentos, manuais, apresentações demonstrando a realização de determinada atividade, entre outros. Tendo em vista essa 
dificuldade, as companhias que conseguem transformar o conhecimento tácito em conhecimento coletivo geram maior vantagem competitiva frente aos competidores.

Outro fator importante abordado por Bohn (1994) é que os funcionários tendem a mudar de cargos, receber propostas em outras companhias e até mesmo se aposentarem. Consequentemente, esses funcionários levam seus conhecimentos, deixando uma lacuna na organização.

A teoria de Nonaka e Takeuchi (1995) é abordada na maioria dos artigos analisados, fazendo menção à metodologia SECI (Socialização, Externalização, Combinação e Internalização). Porém, conforme Hall \& Andriani (2003), não é possível codificar o conhecimento tácito, argumentando que, se esse conhecimento não pode ser codificado, como poderá ser comunicado? Não é sendo possível o gerenciamento desse tipo de conhecimento.

Já, Reychav \& Weisberg (2009), que também adotam a metodologia SECI para a gestão do conhecimento tácito, afirmam que é um processo tipicamente acionado pelo diálogo entre os empregados, direcionando o diálogo para experiências e criação de novos conhecimentos. Além disso, sem essa união e sem a afinidade entre os empregados, torna-se impossível a transferência de conhecimento.

Com essa socialização, Hau et al. (2013) estudam o comportamento dos indivíduos em querer compartilhar o conhecimento. Os autores afirmam que, sem essa intenção do pessoal envolvido na socialização, não haverá transferência de conhecimento, tanto tácito quanto explícito, pois o ser humano tende a reter toda a informação quando se sente ameaçado em sair de sua zona de conforto. Essas ameaças podem ser caracterizadas como: o desligamento da empresa, a troca indesejada do empregado de um departamento para outro, entre outros meios de deslocamento.

Pode-se considerar a afinidade e comunicação entre os empregados um dos principais fatores críticos de sucesso (FCS), pois sem a união destes dois temas, ocasionará omissão de informações essenciais à companhia. Por este motivo, a figura de liderança acaba também se tornando um FCS, pois suas decisões são impactantes e geralmente aceitas por todos os empregados (OYEMOMI et al., 2016).

Já Tyagi et al. (2015) afirmam que, com a utilização da metodologia SECl em conjunto a ferramentas do lean manufacturing, é aprimorada a eficiência da criação do conhecimento, melhorando não apenas na geração ou transferência de conhecimento, mas também na aparição de novos projetos e na melhoria na velocidade na tomada de decisões.

Outros estudos mostram o desenvolvimento de softwares e frameworks baseados na metodologia SECl, conforme ilustrado pela Figura 5. 


\section{Figura 5}

Teorias por trás de um software

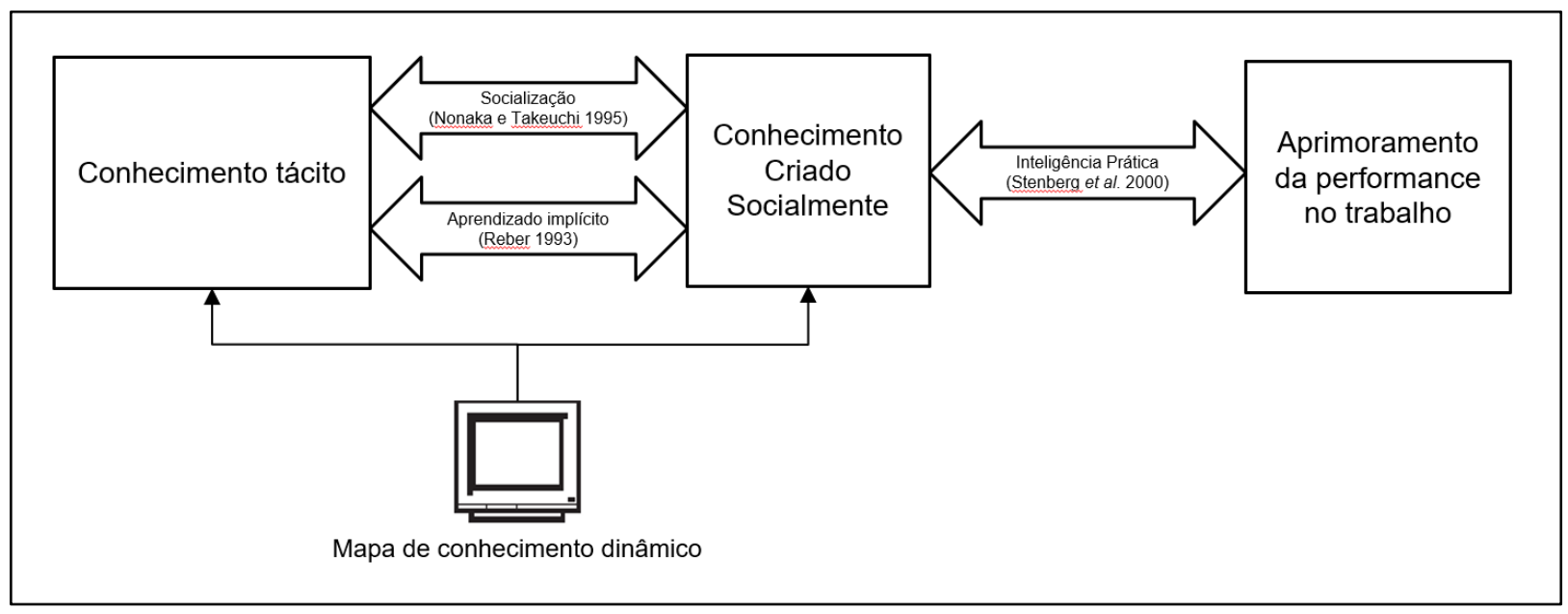

Fonte: Traduzido de Chumg et al. (2015).

Esses softwares, conforme Chumg et al. (2015), podem auxiliar na gestão do conhecimento, mas ainda é dependente da interação humana. Sem a efetiva socialização não é possível explicitar o conhecimento tácito dos indivíduos, que está diretamente relacionado com a parte afetiva entre um funcionário e outro, sendo que, em casos negativos de interação, pode ocorrer a retenção de conhecimento sem o consentimento da empresa, fazendo com que gaste recursos na gestão do conhecimento sem estar ocorrendo.

Para Tserng \& Lin (2004), Lin (2008) e Lin \& Lee (2012), o software desenvolvido é utilizado para a comunicação entre engenheiros mais experientes e novatos do ramo de construção civil. Tal prática é adotada para a prevenção de erros que podem ocorrer durante o desenvolvimento de um novo projeto liderado por um engenheiro júnior. Os autores ainda e afirmam que a maior perda das construtoras é o know-how dos engenheiros experientes, que não são gerenciados adequadamente. O software foi idealizado a fim de criar essa interação entre engenheiros juniors e seniors, com uma interface de comunicação e criar uma base de dados de todo o conhecimento compartilhado entre eles, que uma vez arquivado, estará disponível para quaisquer partes consultarem o conhecimento explicitado.

Para auxiliar na tomada de decisões, também podem ser utilizados mapas cognitivos para formalização, reutilização e posteriormente adaptação do conhecimento tácito no momento de construção dos softwares (NOH et al., 2000).

Zhao et al. (2014) propõem a transferência de conhecimento por intermédio de treinamentos e definem uma estrutura hierárquica seguida de uma estrutura de transferência de conhecimento, conforme ilustrado na figura 6. 
Figura 6

Pirâmide hierárquica e estrutura de transferência de conhecimento

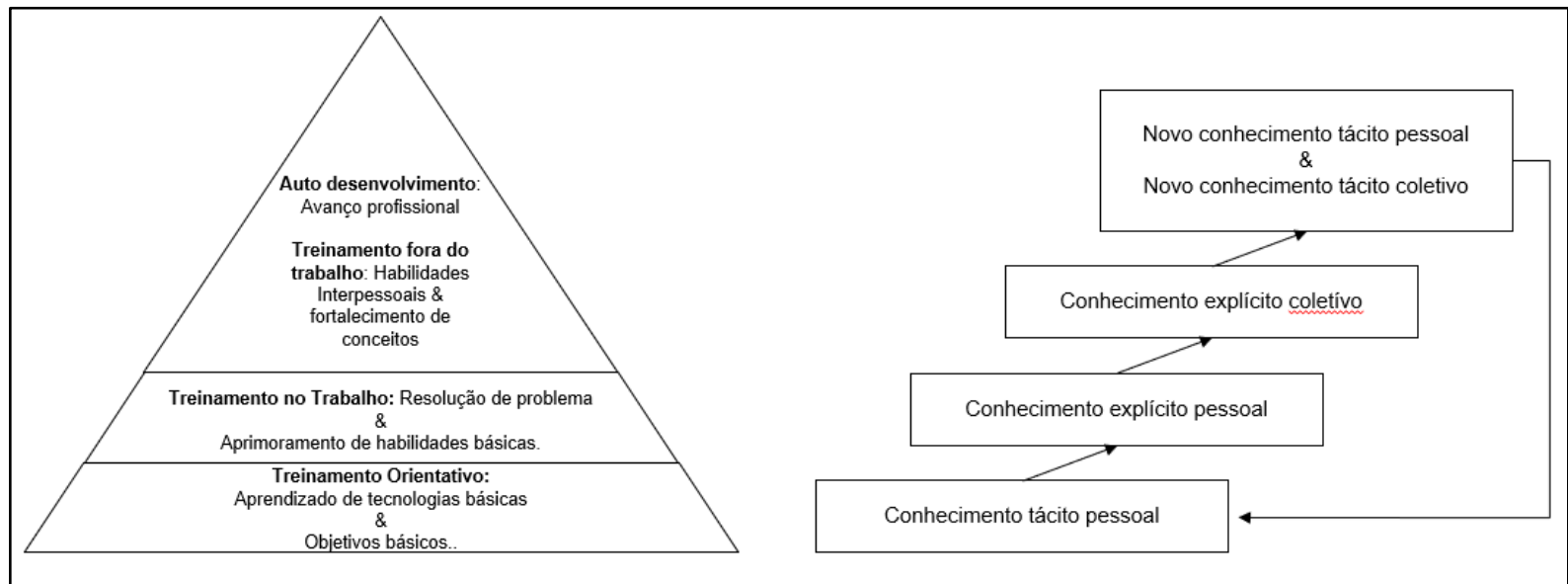

Fonte: Traduzido de Zhao et al. (2014).

Zhao et al. (2014) afirmam que o conhecimento tácito primeiramente se transforma em um conhecimento explícito coletivo devido a socialização e, futuramente, se transforma em tácito individual novamente e, nem sempre, o conhecimento tácito é gerado igualmente, pois, como se trata de sentimentos, é um conhecimento variável de pessoa a pessoa.

De acordo com Desouza (2003), as melhores práticas para explicitação do conhecimento estão em ambientes lúdicos, ou seja, salas de jogos. O autor cita cases de sucesso em duas empresas multinacionais, Unilever e Honda, destacando que a gestão do conhecimento deve partir de uma abordagem top-down, em que a gerência deve fazer mais do que apenas visualizar a gestão do conhecimento, deve incentivar a prática e apoiar a cultura de compartilhamento de conhecimento.

Feng et al. (2017) trazem a proposta de um sistema de incentivo de compartilhamento de conhecimento tácito, porém não é possível concluir essa estruturação devido ao tempo de desenvolvimento. O fator tempo se torna um limitador para a finalização do trabalho.

Já Scaringella e Burtschell (2017) ressaltam a importância de reconhecer, por meio de prêmios ou afins, as pessoas envolvidas no sistema de gestão de conhecimento.

Outra abordagem adotada por Maravilhas \& Martins (2019) é a inserção de pessoas em projetos utilizando novas tecnologias e inovações, as quais são adquiridas por meio de práticas intercompany taxadas como boas práticas. Outra premissa adotada por Belay et al. (2016) é que as pessoas precisam aprender a compartilhar o conhecimento, pois sistemas são utilizados apenas para memorizar as informações. O fator crítico de sucesso está nas pessoas saberem se expressar e na boa comunicação entre elas, porém, de acordo com Scaringella e Burtschell (2017), a transferência de conhecimento será falha se não houver um grau de similaridade entre as pessoas envolvidas na gestão, como por exemplo, o grau de absorção de informação do indivíduo. 
Xiang et al. (2013) propõem a utilização de patentes como forma de transferência do conhecimento tácito, porém há uma limitação que impede o avanço do estudo, o fator de medição. É realizada apenas uma reflexão parcial dos resultados pois, conforme afirmam os autores, não há meio de medição desse tipo de abordagem sem contato pessoal entre receptor e transmissor do conhecimento tácito.

Serna et al. (2017) tratam três tipos de abordagens: interação entre pessoas, utilização de inteligência artificial em sistemas informatizados e métodos associados a técnicas de dinâmica de jogos ou gamificação. Entretanto, o autor não determina a melhor forma de explicitação que deve ser adotada para criação de melhor gestão de conhecimento.

\subsection{Achados via método "bola de neve"}

Após o levantamento dos 21 artigos, apresentados nas seções anteriores, foi possível realizar uma análise detalhada das referências desses artigos por meio do método "bola de neve" (Wohlin, 2014; Contandriopoulos et al., 2010). Isso permite identificar novas possibilidades de achados ou lacunas.

De acordo com Ipe (1998), Dhanaraj et al. (2004) e Reychav \& Weisberg (2010), o conhecimento tácito, além de ser mais difícil de ser compartilhado entre os empregados, seu custo é muito mais elevado, levando em consideração o alto tempo de transferência e a demanda de grandes esforços para o sucesso dessa transferência. Já Liu et al. (2014) enfatizam que a criação de novos conhecimentos acontece de acordo com cada empregado, sem tempo determinado para acontecer, deixando aberto o ciclo de geração de novos conhecimentos, impossibilitando a criação de um indicador temporal para a finalização do ciclo de transferência de conhecimento.

Com base em Chow e Chan (2008) e Lin (2007), as fases sociais da gestão do conhecimento, o compartilhamento do conhecimento consiste em interações entre os empregados. De acordo com Nahapiet \& Ghoshal (1998), tais interações são influenciadas pelo relacionamento afetivo entre os indivíduos, obtendo fluidez no processo de explicitação entre as pessoas com maior grau de afinidade, bem como é sugerido por Yu et al. (2013), que a gestão de conhecimento deve ser mais "humanizada", dando mais atenção para a afinidade entre as pessoas nos times, assim, motivando os integrantes a compartilharem conhecimento, especialmente, o conhecimento tácito.

Já Obrenovic et al. (2014) afirmam que a transferência de conhecimento tácito tem impacto positivo no desempenho dos times, apontando que é mais efetiva dentre os que compartilham o conhecimento tácito livremente, bem como práticas de mentoring, que são eficazes devido ao alto tempo de socialização entre mentor e aprendiz. Outro fator importante apontado por esses autores é 
que, com o advento das novas tecnologias, o tácito pode ser facilmente adquirido por intermédio de mídias, ou seja, em alguns casos, vídeos explicativos que são encontrados em abundância na internet.

\section{Conclusão}

O objetivo deste trabalho foi atingido ao selecionar-21 artigos pelo método de RSL e mais 7 artigos pelo método "bola de neve", no período entre 1998 e 2019, referente às práticas de explicitação do conhecimento tácito.

Foram destacados 4 tipos de práticas possíveis de explicitação de conhecimento: convivência/socialização, softwares, salas/espaços, treinamentos.

Foi possível evidenciar que, dentre os 4 tipos, a convivência/socialização se destaca, ficando clara a necessidade de contato entre as pessoas para se explicitar qualquer tipo de conhecimento. Mesmo com o uso de novas tecnologias, o contato ainda é necessário. Práticas de mentoring se sobressaem às outras práticas, pois se tem uma socialização e convivência mais frequente, mostrando resultados mais eficazes. Destaque-se que esta prática tem um custo mais elevado, pois todos os recursos gastos na gestão têm impacto apenas no mentor e no aprendiz.

Observou-se também outro tipo de explicitação, o desenvolvimento de softwares para auxílio na gestão do conhecimento, mas que ainda dependem da interação humana para o sucesso, tendo a necessidade de as pessoas concordarem e se sentirem confortáveis na fase de socialização. Mesmo com as tecnologias ou softwares mais avançados, é impossível de se obter o conhecimento de uma pessoa que não esteja confiante em relatar todas as suas experiências e vivências, evidenciando ser um grande limitador para o desenvolvimento da gestão de conhecimento. Isso pode trazer resultados indesejáveis para a transferência do conhecimento, tais como, transferência de conhecimentos desnecessários, gastos de recursos e esforços com pessoas desmotivadas ou que se sintam ameaçadas pelo sistema de gestão do conhecimento. Ou seja, a boa comunicação, afinidade e a vontade dos indivíduos em compartilhar e socializarem, sem se sentirem ameaçados em relação à sua zona de conforto, é um fator crítico de sucesso para a externalização do conhecimento tácito.

Outros dois tipos de explicitação observados são as práticas de socialização por meio de treinamentos teóricos ou jogos lúdicos, como mediador/transmissor de conhecimento tácito, nos quais o instrutor poderá expressar seus sentimentos e vivência durante o desenvolvimento de um jogo/aula e salas, ou ambientes, que permitam a facilidade e fluidez no compartilhamento de experiências de determinados assuntos contemplados na gestão de conhecimento tácito.

Em relação às lacunas sobre o tema, foi possível evidenciar o alto tempo gasto para conclusão de estudo de casos, pois o conhecimento tácito, por se tratar de experiência, além da transferência inicial que ocorre durante a socialização entre as partes interessadas, exige testes práticos daquele 
conhecimento adquirido e validado por meio do conhecimento coletivo gerado. Sendo assim, o tempo deste ciclo é alto, obtendo-se apenas resultados parciais dos artigos analisados. Também não foi possível obter um formato padrão de indicadores de desempenho da gestão de conhecimento tácito, já que este tipo de conhecimento pode variar de pessoa para pessoa, tendo em vista a percepção e sentimento individual, o que exige formas diferentes de avaliação.

Posto isto, fica demonstrada a relevância desse estudo no sentido de identificar, de modo agrupado, as quatro principais práticas de explicitação de conhecimento e suas possíveis interações. Não há indicativos de um formato padronizado e único para programas de explicitação e nem indicadores específicos.

Em termos de resultados acadêmicos, destaque-se a identificação destas quatro práticas preponderantes, portanto, qualquer iniciativa de elaboração teórico-conceitual terá maior êxito ao englobá-las todas harmonicamente ou, pelo menos, considerar a primeira delas convivência/socialização - como primordial, dependendo da análise ou da aplicação. Não ficou evidenciado nenhum formato específico ou estrutura básica de programas de explicitação de conhecimento e nem padronização de indicadores de acompanhamento.

No tocante a resultados gerenciais, ficou evidenciado que há a necessidade de certo espaço de tempo para se obter resultados aplicáveis em programas de explicitação de conhecimento, mas que é factível realizá-los, desde que se tenha boa gestão dos recursos utilizados e dos envolvidos (mentores e aprendizes). Será preciso elaborar e acompanhar indicadores apropriados para essa gestão.

A delimitação dos resultados desse estudo está no contexto do recorte temporal (até abril/2019), na consideração de publicações somente do tipo artigo e por considerar apenas 3 bases de dados.

Assim, com o resultado desta RSL, é sugerido que as pesquisas futuras para este tema sejam atualizadas no recorte temporal, considerando outros tipos de publicações (proceedings, por exemplo) e mais bases de dados (IEEE, por exemplo), além de se ampliar as strings de busca para tentar chegar a um possível conjunto mínimo de indicadores necessários para validar a transferência do conhecimento. Também, seria relevante, a realização de estudos empíricos de gestão do conhecimento tácito, no que tange à sua efetividade ao final do programa.

\section{Agradecimentos}

O presente trabalho foi realizado com apoio da Coordenação de Aperfeiçoamento de pessoal de Nível Superior - Brasil (CAPES) - Código de Financiamento 001. 


\section{Referências}

AL-TABBAA, O \& ANK AH, S. (2016). Social capital to facilitate 'engineered' university-industry collaboration for technology transfer: A dynamic perspective. Technological Forecasting \& Social Change, n.104, p.1-15.

ARLING, P. A.; CHUN, M. W. S. (2011). Facilitating new knowledge creation and obtaining KM maturity. Journal of Knowledge Management, 15, 231-250.

AUGIER, M. \& VENDELO, M.T. (1999). Networks, Cognition and management of tacit knowledge. Journal of Knowledge Management, 3, 4, 252-61.

BADARACCO JR JL. (1991). Alliances speed knowledge transfer. Plann Rev.,19 (2):10-6.

BEAL, A. (2014). Gestão estratégica da informação. 1. Ed. São Paulo: Atlas.

BELAY, A. M., TORP, O. \& THODESEN, C. (2016). Managing concurrent construction projects using knowledge management and set-based thinking. CCC, 25-28 June.

BOHN, R. (1994). Measuring and managing technological knowledge. Sloan Manag. Rev., 36(1), 61-73.

$\mathrm{CHOI}, \mathrm{B}$. \& LEE, H. (2002). Knowledge management strategy and its link to knowledge creation process. Expert Systems with Applications, 23(3), 173-187.

CHOI, B.; POON, K. S.; DAVIS, G. J. (2008). Effects of knowledge management strategy on organizational performance: a complementarity theory-based approach. OMEGA, The International Journal of Management Science, v. 36, p. 235-251.

CHOO, C. W. \& NETO, R. C. D. A. (2010). Beyond the ba: managing enabling contexts in knowledge organizations. Journal of Knowledge Management, Vol. 14 Iss 4 pp. $592-610$.

CHOW, W. S. \& CHAN, L. S. (2008). Social network, social trust, and shared goals in organizational knowledge sharing. Information and Management, 45, 458-465.

CHUMG, H. F., COOKE, L., FRY, J. \& HUNG, I. H. (2015). Factors affecting knowledge sharing in the virtual organization: Employees' sense of well-being as a mediating effect. Computers in Human Behavior 44, 70-80.

COLLINS, H. (2007). Bicycling on the moon: Collective tacit knowledge and somatic-limit tacit knowledge. Organization Studies, 28(2), 257-262.

CONTANDRIOPOULOS, D., LEMIRE, M., DENIS, J. L., TREMBLAY, E. (2010). Knowledge Exchange Processes in Organizations and Policy Arenas: A Narrative Systematic Review of the Literature. The Milbank Quarterly, Vol. 88, No. 4 (pp. 444-483).

CUMMING, J. N. (2004). Work groups, structural diversity, and knowledge sharing in a global organization. Management Science. 50(3), 352-364.

DESOUZA, K. C. (2003). Strategic contributions of game rooms to knowledge management: some preliminary insights. Information \& Management 41, 63-74. 
DHANARAJ, C., LYLES, R. A., STEENSMA, H. K. \& TIHANYI, L. (2004). Managing tacit and explicit knowledge transfer in IJVs: The role of relational embeddedness and the impact on performance. Journal of International Business Studies, 35, 428-442.

DUTTA, S. (1997). Strategies for implementing knowledge-based systems. IEEE Transactions on Engineering Management, 44 (1), 79-90.

FENG, W. J., YAN, C. M., JIE, F. L. \& JU, Y. J. (2017). The Construction of Enterprise Tacit Knowledge Sharing Stimulation System Oriented to Employee Individual. Procedia Engineering 174, 289 300.

GILL, S.P. (2000). The tacit dimension of dialogue for knowledge transfer. Helsinki School of Economics and Business Administration.

HALL, R. \& ANDRIANI, P. (2003). Managing knowledge associated with innovation. Journal of Business Research 56, 145-152.

HAU, Y. S., KIM, B., LEE, H. \& KIM, Y. G. (2013). The effects of individual motivations and social capital on employees' tacit and explicit knowledge sharing intentions. International Journal of Information Management 33, 356-366.

HOOD, W. W. \& WILSON, C. S. (2001). The literature of bibliometrics, and informetrics scientometrics. Scientometrics, v.52, n.2, p.291-314.

IPE, M. (1998). Knowledge sharing in organizations: A conceptual framework. Human Resource Development Review, 2, 337-359.

JESSON, J., MATHESON, L. \& LACEY, F. M. (2011). Doing your literature review traditional and systematic techniques. Sage: California.

KAKABADSE, N. K., KOUZMIN, A. \& KAKABADSE, A. (2001). From tacit knowledge to knowledge management: leveraging invisible assets. Knowl. Process. 8, 137-154.

KITCHENHAM, B. (2007). Guidelines for performing systematic literature review in software engineering. EBSE Technical Report - version 2.3. Keele University and University of Durham, UK. 9 July.

KUHN, T. S. (1996 [1962]). The structure of scientific revolutions. Chicago: University of Chicago Press.

LEON, R. D.; RODRÍGUEZ-RODRÍGUEZ, R.; GÓMEZ-GASQUET, P.; MULA, J. (2017). Social network analysis: A tool for evaluating and predicting future knowledge flows from an insurance organization. Technological Forecasting and Social Change, v. 114, p. 103-118.

LIN, H. F. (2007). Effects of extrinsic and intrinsic motivation on employee knowledge sharing intentions. Journal of Information Science, 33, 135-149.

LIN, Y. C. (2008). Developing construction assistant experience management system using peoplebased maps. Automation in Construction, 17, 975-982.

LIN, Y. C. \& LEE, H. Y. (2012). Developing project communities of practice-based knowledge management system in construction. Automation in Construction 22, 422-432. 
LIU, S., MOIZER, J., MEGICKS, P., KASTURIRATNE, D. \& JAYAWICKRAMA, U. A. (2014). Knowledge chain management framework to support integrated decisions in global supply chains. Prod. Plann. Control, 25(8), 639-649.

MARAVILHAS, S. \& MARTINS, J. (2019). Strategic knowledge management in a digital environment: Tacit and explicit knowledge in Fab Labs. Journal of Business Research 94, 353-359.

MACHADO, A. C. M. \& DESIDERI, P. E. S. (2002). As dificuldades na difusão do conhecimento tácito nas organizações. XXII Encontro Nacional de Engenharia de Produção, Curitiba-PR.

NAHAPIET, J. \& GHOSHAL, S. (1998). Social capital, intellectual capital and the organizational advantage. Academy of Management Review, 23, 242-266.

NOH, J.B., LEE, K.C., KIM, J.K., LEE, J.K. \& KIM, S.H. (2000). A case-based reasoning approach to cognitive map-driven tacit knowledge management. Expert Systems with Applications, 19, 249-259.

NONAKA, I., KODAMA, M., HIROSE, A. \& KOHLBACHER, F. (2014). Dynamic fractal organizations for promoting knowledge-based transformation - A new paradigm for organizational theory. European Management Journal, Elsevier, 32, 137-146.

NONAKA, I. \& TAKEUCHI, H. (1995). The Knowledge-Creating Company. New York: Oxford University Press.

NONAKA, I.; TAKEUCHI, H. (2008) Teoria da criação do conhecimento organizacional. In: TAKEUCHI, H.; NONAKA, I. Gestão do conhecimento. Porto Alegre: Bookman, 54-90.

OBRENOVIC, B., OBRENOVIC, S. \& HUDAYKULOV, A. (2014). The value of knowledge sharing: impact of tacit and explicit knowledge sharing on team performance of scientists. Information and Knowledge Management. Vol.4, No.4.

OLAISEN, J. \& REVANG, O. (2018). Exploring the performance of tacit knowledge: How to make ordinary people deliver extraordinary results in teams. International Journal of Information Management 43, 295-304.

OYEMOMI, O., LIU, S., NEAGA, I. \& ALKHURAIJI, A. (2016). How knowledge sharing and business process contribute to organizational performance: Using the fs $Q C A$, Journal of Business Research 69, 5222-5227.

POLANYI, M. (1969). The logic of tacit inference. In M. Grene (Ed.), Knowing and Being:Essays by Michael Polanyi (pp.140- 44.Chicago:University of Chicago Press.Polanyi, M. (1983 [1966]).The tacit dimension.London:Routledge \& Kegan Paul.

PULIC, A. (1998). Measuring the performance of intellectual potential in the knowledge economy. 19th Annual National Business Conference/Carver Tiffany, Hamilton, Stahlke, J. (Ed.), Ontario.

RAMY, A., FLOODY, J., RAGAB, M. A. F. \& ARISHA, A. (2018). A scientometric analysis of Knowledge Management Research and Practice literature: 2003-2015. Knowledge Management Research \& Practice, v.16, n.1, p. 66-77. 
REYCHAV, I. \& WEISBERG, J. (2009). Going beyond technology: Knowledge sharing as a tool for enhancing customer-oriented attitudes. International Journal of Information Management, 29(5), 353-361.

REYCHAV, I. \& WEISBERG, J. (2010). Bridging intention and behavior of knowledge sharing. Journal of Knowledge Management, 14(2), 285-300.

RIBEIRO, R. (2012). Tacit Knowledge Management. In Evan, S., Stone, D. and Feist, Greg (ed.) Tacit Knowledge: New Theories and Practices: Special Issue of Phenomenology and the Cognitive Sciences.

SCARINGELLA, L.; BURTSCHELL, F. (2017). The challenges of radical innovation in Iran: Knowledge transfer and absorptive capacity highlights - Evidence from a joint venture in the construction sector. Technological Forecasting \& Social Change, 122, 151-169.

SERNA, E. M., BACHILLER, O. S. \& SERNA, A. A. (2017). Knowledge meaning and management in requirements engineering. International Journal of Information Management, 37, 155-161.

SZULANSKI, G. (1996). Exploring internal stickiness: impediments to the transfer of best practice within the firm. Strategy Manage J; 17:27-43.

TEECE, D.J. and AL-AALI, A. (2011). Knowledge assets, capabilities, and the theory of the firm. Handbook of Organizational Learning and Knowledge Management, Vol. 2, pp. 505-534.

TRANFIELD, D., DENYER, D. \& SMART, P. (2003). Towards a methogology for developing evidenceinformed management knowledge by means of systematic review. Brithish Journal of Management. V. 14, p. 207-222.

TSERNG, H.P. \& LIN, Y.C. (2004). Developing an activity-based knowledge management system for contractors. Automation in Construction, 13, 781- 802.

TYAGI, S., CAI, X., YANG, K. \& CHAMBERS, T. (2015). Lean tools and methods to support efficient knowledge creation. International Journal of Information Management, 35, 204-214.

TURBAN, E., SHARDA, R., ARONSON, J.E. \& KING, D. (2009). Business intelligence: um enfoque gerencial para a inteligência do negócio. Porto Alegre: Bookman.

WAGNER, R. K. \& STERNBERG, R. J. (1985). Practical intelligence in real world pursuits: the role of tacit knowledge. Journal of Personality and Social Psychology, 48, 436-458.

WIIG, K. M. (1993). Knowledge management foundations: Thinking about thinking - how people and organizations create, represent, and use knowledge. Arlington. TX: Schema Press.

WITTGENSTEIN, L. (1976). Philosophical investigations. Oxford: Blackwell.

WOHLIN, C. (2014). Guidelines for Snowballing in Systematic Literature Studies and a Replication in Software Engineering. EASE '14, May 13 - 14 2014, London, England, BC, United Kingdom.

WOO, J.H., CLAYTON, M. J., JOHNSON, R. E., FLORES, B. E. \& ELLIS, C. (2004). Dynamic Knowledge Map: reusing experts' tacit knowledge in the AEC industry. Automation in Construction, 13, 203207. 
XIANG, X. Y., CAI, H., LAM, S. \& PEI, Y. L. (2013). International knowledge spillover through co-inventors: An empirical study using Chinese assignees' patent data. Technological Forecasting \& Social Change 80, 161-174.

YU, Y., HAO, J. X., DONG, X. Y. \& KHALIFA, M. (2013). A multilevel model for effects of social capital and knowledge sharing in knowledge-intensive work teams. International Journal of Information Management, 33, 780- 790.

ZHANG, X.; JIANG, J.Y. (2015). With whom shall I share my knowledge? A recipient perspective of KS. Journal of Knowledge Management, Vol. 19 No. 2, pp. 277-295.

ZHAO, J., QI, Z. \& PABLOS, P. O. (2014). Enhancing enterprise training performance: Perspectives from knowledge transfer and integration. Computers in Human Behavior 30, 567-573. 